\title{
A fixed point theorem for Meir-Keeler contractions in ordered metric spaces
}

\author{
Jackie Harjani, Belén López and Kishin Sadarangani*
}

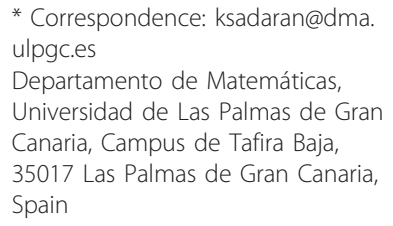

* Correspondence: ksadaran@dma. ulpgces

Departamento de Matemáticas, Universidad de Las Palmas de Gran Canaria, Campus de Tafira Baja, 35017 Las Palmas de Gran Canaria, Spain

\section{Abstract}

The purpose of this paper is to present some fixed point theorems for Meir-Keeler contractions in a complete metric space endowed with a partial order.

MSC: $47 \mathrm{H} 10$.

Keywords: fixed point, ordered metric spaces, Meir-Keeler contraction

\section{Introduction and preliminaries}

The Banach contraction mapping principle is one of the pivotal results of analysis. It is widely considered as the source of metric fixed point theory. Also, its significance lies in its vast applicability in a number of branches of mathematics.

Generalization of the above principle has been a heavily investigated branch of research. In particular, Meir and Keeler [1] present the following fixed point theorem.

Theorem 1.1. [1] Let $(X, d)$ be a complete metric space and $T: X \rightarrow X$ an operator. Suppose that for every $\varepsilon>0$ there exists $\delta(\varepsilon)>0$ such that for $x, y \in X$

$$
\varepsilon \leq d(x, y)<\varepsilon+\delta(\varepsilon) \Rightarrow d(T x, T y)<\varepsilon .
$$

Then, $T$ admits a unique fixed point $\xi \in X$ and for any $x \in X$, the sequence $\left\{T^{n} x\right\}$ converges to $\xi$.

The purpose of this article is to present a version of Theorem 1.1 in the context of ordered metric spaces.

Existence of fixed point in partially ordered sets has been recently studied in [2-20].

In the context of ordered metric spaces, the usual contraction is weakened but at the expense that the operator is monotone.

\section{Fixed point results: nondecreasing case}

Our starting point is the following definition.

Definition 2.1. Let $(X, \leq)$ be a partially ordered set and $T: X \rightarrow X$ a mapping. We say that $T$ is nondecreasing if for $x, y \in X$

$$
x \leq y \Rightarrow T x \leq T y .
$$

This definition coincides with the notion of a nondecreasing function in the case where $X=\mathbb{R}$ and $\leq$ represents the usual total order in $\mathbb{R}$.

Remark 2.2. The contractive condition given by Meir-Keeler:

\section{Springer}

(C) 2011 Harjani et al; licensee Springer. This is an Open Access article distributed under the terms of the Creative Commons Attribution License (http://creativecommons.org/licenses/by/2.0), which permits unrestricted use, distribution, and reproduction in any medium, provided the original work is properly cited. 
For every $\varepsilon>0$, there exists $\delta(\varepsilon)>0$ such that for $x, y \in X$

$$
\varepsilon \leq d(x, y)<\varepsilon+\delta(\varepsilon) \Rightarrow d(T x, T y)<\varepsilon
$$

implies that the operator $T: X \rightarrow X$ is strictly nonexpansive (this means that for $x, y$ $\in X, x \neq y, d(T x, T y)<d(x, y))$.

Therefore, any operator $T$ satisfying the Meir-Keeler condition is continuous.

In what follows, we present the following theorem which is a version of Theorem 1.1 in the context of ordered metric spaces when the operator is nondecreasing.

Theorem 2.3. Let $(X, \leq)$ be a partially ordered set and suppose that there exists a metric $d$ in $X$ such that $(X, d)$ is a complete metric space. Let $T: X \rightarrow X$ be a continuous and nondecreasing mapping such that for every $\varepsilon>0$ there exists $\delta(\varepsilon)>0$ satisfying

$$
\varepsilon \leq d(x, y)<\varepsilon+\delta(\varepsilon) \text { and } x<y \Rightarrow d(T x, T y)<\varepsilon .
$$

If there exists $x_{0} \in X$ with $x_{0} \leq T x_{0}$, then $T$ has a fixed point.

Remark 2.4. Condition (??) does not imply that $T$ is continuous as it is proven with the following example.

Let $X=[0,1]$ be the unit interval with the usual metric and the order given by $R=$ $\{(x, x): x \in X\}$. Consider the operator $T: X \rightarrow X$ given by $T x=0$ if $x \neq 1$ and $T x=1$ if $x=1$.

Obviously, $T$ is not continuous and, as the elements in $X$ are only comparable to themselves, $T$ satisfies (??).

This shows that the continuity of $T$ in Theorem 2.3 is not redundant (compare with Remark 2.2 and Theorem 1.1).

Remark 2.5. Condition (1) does not imply that $T$ is strictly nonexpansive but $d(T x$, $T y)<d(x, y)$ is true if $x<y$ as it can be seen using $\varepsilon=d(x, y)$ in (1) when $x<y$.

Proof of Theorem 2.3. If $T x_{0}=x_{0}$, then the proof is finished.

Suppose that $x_{0}<T x_{0}$ and $T$ is a nondecreasing mapping, we obtain by induction that

$$
x_{0}<T x_{0} \leq T^{2} x_{0} \leq T^{3} x_{0} \leq \cdots \leq T^{n} x_{0} \leq T^{n+1} x_{0} \leq \cdots .
$$

Put $x_{n+1}=T^{n} x_{0}$. Obviously, $\left(x_{n}\right)$ is a nondecreasing sequence.

For better readability, we divide the proof into several steps.

Step 1: $\lim _{n \rightarrow \infty} d\left(x_{n}, x_{n+1}\right)=0$.

In fact, if the sequence $\left(x_{n}\right)$ is not strictly nondecreasing, then we can find $n_{0} \in \mathbb{N}$ such that $x_{n_{0}}=x_{n_{0}+1}$ and the result follows. If $\left(x_{n}\right)$ is strictly nondecreasing, then by Remark 2.5, $\left(d\left(x_{n}, x_{n+1}\right)\right)$ is strictly decreasing, and hence, it is convergent. Put $r=\lim _{n}$ $\rightarrow \infty d\left(x_{n}, x_{n+1}\right)$ (notice that $r=\inf \left\{d\left(x_{n}, x_{n+1}\right): n \in \mathbb{N}\right\}$ ).

Now, we will prove that $r=0$.

Suppose that $r>0$.

Applying condition (??) to $r>0$ we can find $\delta(r)>0$ such that

$$
\left.\begin{array}{c}
r \leq d(x, y)<r+\delta(r) \\
\text { and } x<y
\end{array}\right\} \Rightarrow d(T x, T y)<r .
$$

Since $r=\lim _{\mathrm{n} \rightarrow \infty} d\left(x_{n}, x_{n+1}\right)$, there exists $n_{0} \in \mathbb{N}$ such that $r \leq d\left(x_{n_{0}}, x_{n_{0}+1}\right)<r+\delta(r) \quad$ and, as $\quad x_{n_{0}}<x_{n_{0}+1}$, we have $d\left(T x_{n_{0}}, T x_{n_{0}+1}\right)=d\left(x_{n_{0}+1}, x_{n_{0}+2}\right)<r$.

This is a contradiction because $r=\inf \left\{d\left(x_{n}, x_{n+1}\right): n \in \mathbb{N}\right\}$. 
Therefore, $r=0$. This means that

$$
\lim _{n \rightarrow \infty} d\left(x_{n}, x_{n+1}\right)=0
$$

Step 2: $\left(x_{n}\right)$ is a Cauchy sequence.

In fact, fix $\varepsilon>0$ and arbitrary.

By condition (??), there exists $\delta(\varepsilon)>0$ (which can be choosen satisfying $\delta(\varepsilon) \leq \varepsilon$ ) such that

$$
\varepsilon \leq d(x, y)<\varepsilon+\delta(\varepsilon) \text { and } x<y \Rightarrow d(T x, T y)<\varepsilon
$$

On the other hand, by (??), there exists $n_{0} \in \mathbb{N}$ such that

$$
d\left(x_{n-1}, x_{n}\right)<\delta(\varepsilon) \text { for any } n>n_{0} .
$$

Fix $n>n_{0}$ and in order to prove that $\left\{x_{n}\right\}$ is a Cauchy sequence it is sufficient to show that

$$
d\left(x_{n}, x_{n+p}\right) \leq \varepsilon \quad \text { for } p=1,2,3, \ldots
$$

We will use mathematical induction.

For $p=1$ by (??) and the fact that $\delta(\varepsilon) \leq \varepsilon$ we obtain

$$
d\left(x_{n}, x_{n+1}\right)<\delta(\varepsilon) \leq \varepsilon .
$$

Now, we assume that (??) holds for some fixed $p$.

Then, using (??) and the inductive hypothesis, we get

$$
d\left(x_{n-1}, x_{n+p}\right) \leq d\left(x_{n-1}, x_{n}\right)+d\left(x_{n}, x_{n+p}\right)<\delta(\varepsilon)+\varepsilon .
$$

Now, we consider two cases.

Case 1: $d\left(x_{n-1}, x_{n+p}\right) \geq \varepsilon$.

In this case, taking into account (??),

$$
\varepsilon \leq d\left(x_{n-1}, x_{n+p}\right)<\varepsilon+\delta(\varepsilon)
$$

and, since $x_{n-1}<x_{n+p}$, by (??)

$$
d\left(T x_{n-1}, T x_{n+p}\right)=d\left(x_{n}, x_{n+p+1}\right)<\varepsilon .
$$

This proves that (??) is satisfied by $p+1$.

Case 2: $d\left(x_{n-1}, x_{n+p}\right)<\varepsilon$.

As $d\left(x_{n-1}, x_{n+p}\right)>0$ (because $\left\{x_{n}\right\}$ is a nondecreasing sequence and $d\left(x_{n}, x_{n+1}\right)>0$ for any $n=0,1,2, \ldots)$, applying condition (??) for $\varepsilon_{0}=d\left(x_{n-1}, x_{n+p}\right)$ we can get

$$
d\left(T x_{n-1}, T x_{n+p}\right)=d\left(x_{n}, x_{n+p+1}\right)<d\left(x_{n-1}, x_{n+p}\right)<\varepsilon
$$

(notice that $x_{n-1}<x_{n+p}$ ) and this proves that (??) is satisfied by $p+1$.

Therefore, $\left\{x_{n}\right\}$ is a Cauchy sequence.

Since $X$ is a complete metric space, there exists $z \in X$ such that $\lim _{n \rightarrow \infty} x_{n}=z$. Finally, the continuity of $T$ implies that

$$
z=\lim _{n \rightarrow \infty} x_{n+1}=\lim _{n \rightarrow \infty} T x_{n}=T z
$$

and, therefore, $z$ is a fixed point of $T$. 
This finishes the proof.

In what follows we prove that Theorem 2.3 is still valid for $T$ not necessarily continuous, assuming the following hypothesis:

$$
\text { if }\left(x_{n}\right) \text { is a nondecreasng sequence such that } x_{n} \rightarrow x \text {, then }
$$

there exists a subsequence $\left(x_{n(k)}\right)$ of $\left(x_{n}\right)$ such that $x_{n(k)} \leq x$ for all $k \in \mathbb{N}$

Theorem 2.6. If in Theorem 2.3 we replace the continuity of $T$ by condition (??) the result is true.

Proof. Following the proof of Theorem 2.3, we only have to check that $T z=z$.

As $\left(x_{n}\right)$ is a nondecreasing sequence in $X$ with $x_{n} \rightarrow z$, by condition (??), we can find a subsequence $\left(x_{n(k)}\right)$ such that $x_{n(k)} \leq z$ for all $k \in \mathbb{N}$.

If there exists $k_{0} \in \mathbb{N}$ such that $x_{n\left(k_{0}\right)}=z$, then the nondecreasing character of $\left(x_{n}\right)$ gives us that

$$
x_{k}=z \quad \text { for all } k \geq n\left(k_{0}\right) .
$$

Particularly, $x_{n\left(k_{0}\right)}=z=x_{n\left(k_{0}\right)+1}=T\left(x_{n\left(k_{0}\right)}\right)$, and $x_{n\left(k_{0}\right)}=z$ is a fixed point of $T$.

Suppose that for any $k \in \mathbb{N}, x_{n(k)}<z$.

Applying condition (??) to $\varepsilon=d\left(x_{n(k)}, z\right)$ for $k$ fixed and arbitrary, we have

$$
d\left(T x_{n(k)}, T z\right)=d\left(x_{n(k)+1}, T z\right)<d\left(x_{n(k)}, z\right) .
$$

As $x_{n(k)} \rightarrow z$, the last inequality implies that $x_{n(k)+1} \rightarrow T z$.

As $\left(x_{n(k)+1}\right)$ is a subsequence of $\left(x_{n}\right)$ and $x_{n} \rightarrow z$ we have $x_{n(k)+1} \rightarrow z$.

Now, the uniqueness of the limit in complete metric spaces gives us $T z=z$.

This finishes the proof.

Now, we present an example where it can be appreciated that assumptions in Theorems 2.3 and 2.6 do not guarantee uniqueness of the fixed point.

Let $X=\{(1,0),(0,1)\} \subset \mathbb{R}^{2}$ and consider the usual order

$$
(x, y) \leq(z, t) \Leftrightarrow x \leq z \text { and } \mathrm{y} \leq \mathrm{t} .
$$

Then, $(X, \leq)$ is a partially ordered set whose different elements are not comparable. Besides, $\left(X, d_{2}\right)$ is a complete metric space considering $d_{2}$ the Euclidean distance. The identity map $T(x, y)=(x, y)$ is trivially continuous and nondecreasing and condition (??) of Theorem 2 is satisfied since elements in $X$ are only comparable to themselves. Moreover, $(1,0) \leq T(1,0)=(1,0)$ and the operator $T$ has two fixed points.

In what follows, we present a sufficient condition for the uniqueness of the fixed point in Theorems 2.3 and 2.6. This condition appears in [15] and says:

For $x, y \in X$, there exists $z \in X$ which is comparable to $x$ and $y$.

Theorem 2.7. Adding condition (??) to the hypotheses of Theorem 2.3 (or Theorem 2.6) we obtain the uniqueness of the fixed point of $T$.

Proof. Suppose that there exist $z, y \in X$ which are fixed points of $T$ and $z \neq y$.

We consider two cases.

Case 1: Suppose that $z$ and $y$ are comparable.

Without loss of generality, we suppose $z<y$. 
Putting $\varepsilon=d(z, y)$ and applying condition (??) of Theorem 1, we get

$$
d(T z, T y)=d(z, y)<\varepsilon=d(z, y)
$$

which is a contradiction.

Case 2: Suppose that $z$ and $y$ are not comparable.

By condition (??), there exists $x \in X$ comparable to $z$ and $y$.

Suppose $z<x$ (the same argument serves for $x<z$ ).

Monotonicity of $T$ implies that $T^{n} z=z \leq T^{n} x$ for $n=1,2,3, \ldots$

We consider two possibilities:

(a) Suppose that there exists $n_{0} \in \mathbb{N}$ such that $T^{n_{0}} z=z=T^{n_{0}} x$. Since $z$ is a fixed point of $T, T^{n} x=z$ for $n \geq n_{0}$, and, consequently, $T^{n} x \rightarrow z$.

(b) Suppose that $T^{n} z=z<T^{n} x$ for any $n=1,2,3, \ldots$ Applying condition (??) of Theorem 1 for $\varepsilon=d\left(T^{n} z, T^{n} x\right)$ (where $n \in \mathbb{N}$ is fixed but arbitrary), we have

$$
d\left(T^{n+1} z, T^{n+1} x\right)=d\left(z, T^{n+1} x\right) \leq d\left(T^{n} z, T^{n} x\right)=d\left(z, T^{n} x\right) .
$$

Thus, $\left\{d\left(z, T^{n} x\right)\right\}$ is a decreasing sequence of positive real numbers and, consequently, there exists $r \geq 0$ such that

$$
\lim _{n \rightarrow \infty} d\left(z, T^{n} x\right)=r
$$

Suppose $r>0$.

Applying condition (??) of Theorem 2.3 for $\varepsilon=r$ we find $\delta(r)>0$ such that

$$
r \leq d(u, v)<r+\delta(r) \text { and } u<v \Rightarrow d(T u, T v)<r .
$$

As $\lim _{n \rightarrow \infty} d\left(z, T^{n} x\right)=r=\inf \left\{d\left(z, T^{n} x\right): n \in \mathbb{N}\right\}$, there exists $n_{0} \in \mathbb{N}$ such that

$$
r \leq d\left(z, T^{n_{0}} x\right)<r+\delta(r)
$$

and, since $z<T^{n_{0}} x$, (??) gives us

$$
d\left(T z, T^{n_{0}+1} x\right)=d\left(z, T^{n_{0}+1} x\right)<r,
$$

which contradicts to $r=\inf \left\{d\left(z, T^{n} x\right): n \in \mathbb{N}\right\}$.

Therefore, $z=y$.

This finishes the proof.

\section{Fixed point results: nonincreasing case}

We start this section with the following definition.

Definition 3.1. Let $(X, \leq)$ be a partially ordered set and $T: X \rightarrow X$. We say that $T$ is nonincreasing if for $x, y \in X$

$$
x \leq y \Rightarrow T x \geq T y .
$$

The main result of this section is the following theorem.

Theorem 3.2. Let $(X, \leq)$ be a partially ordered set satisfying condition (??) and suppose that there exists a metric $d$ in $X$ such that $(X, d)$ is a complete metric space. Let $T$ : $X \rightarrow X$ be a nonincreasing mapping such that for any $\varepsilon>0$ there exists $\delta(\varepsilon)>0$ satisfying

$$
\varepsilon \leq d(x, y)<\varepsilon+\delta(\varepsilon) \text { and } x<y \Rightarrow d(T x, T y)<\varepsilon .
$$


(a) If there exists $x_{0} \in X$ with $x_{0} \leq T x_{0}$ or $x_{0} \geq T x_{0}$, then $\inf \{d(x, T x): x \in X\}=0$.

(b) If, in addition, $X$ is compact and $T$ is continuous, then $T$ has a unique fixed point.

Proof. (a) If $T x_{0}=x_{0}$, then it is obvious that $\inf \{d(x, T x): x \in X\}=0$. Suppose that $x_{0}$ $<T x_{0}$ (the same argument serves for $T x_{0}<x_{0}$ ).

In virtue that $T$ is nonincreasing the consecutive terms of the sequence $\left(T^{n} x_{0}\right)$ are comparable.

Suppose that there exists $n_{0} \in \mathbb{N}$ such that $T^{n_{0}} x_{0}=T^{n_{0}+1} x_{0}$.

In this case, $T\left(T^{n_{0}} x_{0}\right)=T^{n_{0}} x_{0}$ and, consequently, $\inf \{d(x, T x): x \in X\}=0$ and this finishes the proof.

Now, we suppose that $T^{n} x_{0} \neq T^{n+1} x_{0}$ for any $n=1,2, \ldots$

Since $T^{n} x_{0}$ and $T^{n+1} x_{0}$ are comparable applying the contractive condition we obtain

$$
d\left(T^{n+1} x_{0}, T^{n+2} x_{0}\right)<d\left(T^{n} x_{0}, T^{n+1} x_{0}\right)
$$

and this inequality is satisfied by any $n \in \mathbb{N}$.

Thus, $\left\{d\left(T^{n} x_{0}, T^{n+1} x_{0}\right)\right\}$ is a decreasing sequence of positive real numbers and, consequently, $\lim _{n \rightarrow \infty} d\left(T^{n} x_{0}, T^{n+1} x_{0}\right)=r$ for certain $r \geq 0$.

Using a similar argument that in Theorem 2.3, we prove that $r=0$.

Finally, the fact $\lim _{n \rightarrow \infty} d\left(T^{n} x_{0}, T^{n+1} x_{0}\right)=0$ implies that $\inf \{d(x, T x): x \in X\}=0$.

This finishes the proof of (a).

(b) Suppose that $X$ is compact and $T$ is continuous.

Taking into account that the mapping

$$
\begin{gathered}
X \rightarrow \mathbb{R}^{+} \\
x \mapsto d(x, T x)
\end{gathered}
$$

is continuous and the fact that $X$ is compact, we can find $z \in X$ such that

$$
d(z, T z)=\inf \{d(x, T x): x \in X\} .
$$

By $(a), d(z, T z)=\inf \{d(x, T x): x \in X\}=0$ and, therefore, $z$ is a fixed point of $T$.

The uniqueness of the fixed point is proved as in Theorem 2.7.

Remark 3.3. A parallel result in the nonincreasing case cannot be obtained using a similar argument as in Theorem 2.3 because the proof that $\left(x_{n}\right)$ is a Cauchy sequence uses that $x_{n-1}$ and $x_{n+p}$ are comparable and this can be false when $T$ is a nonincreasing operator (see, Theorem 2.3).

\section{Examples}

In this section, we present some examples which illustrate our results.

Example 4.1. Let $X=\{(0,1),(1,0),(1,1)\} \subset \mathbb{R}^{2}$ with the Euclidean distance $d_{2} .\left(X, d_{2}\right)$ is a complete metric space. We consider the order $\leq$ in $X$ given by $R=\{(x, x): x \in X\}$.

Notice that the elements in $X$ are only comparable to themselves. Therefore, condition (??) of Theorem 2.3 is satisfied for any operator $T: X \rightarrow X$.

We consider the operator $T: X \rightarrow X$ defined by

$$
\begin{aligned}
& T(1,0)=(0,1) \\
& T(0,1)=(1,0), \\
& T(1,1)=(1,1) .
\end{aligned}
$$


Obviously, $T$ is a continuous and nondecreasing operator satisfying $(1,1) \leq T(1,1)=$ $(1,1)$.

Theorem 2.3 gives us the existence of a fixed point for $T$ (which is the point $(1,1)$ ).

On the other hand, the operator $T$ does not satisfy Meir-Keeler condition appearing in

Theorem 1.1, because for $\varepsilon=d_{2}((0,1),(1,0))=\sqrt{2}$ the inequality

$$
d_{2}(T(0,1), T(1,0))=d_{2}((1,0),(0,1))=\sqrt{2}<\varepsilon
$$

fails.

Therefore, this example cannot be treated by Theorem 1.1.

Notice that in Example 4.1, we have uniqueness of the fixed point and condition (??) is not satisfied by $(X, \leq)$ (notice that condition (??) fails for the elements $(0,1),(1,0) \in$ $X$ ). This proves that condition (??) is not a necessary condition for the uniqueness of the fixed point.

Example 4.2. Consider the same space $X$ that in Example 4.1 with the Euclidean distance $d_{2}$ and with the order given by

$$
R=\{(x, x): x \in X\} \cup\{((0,1),(1,1))\} .
$$

Consider the operator $T: X \rightarrow X$ given by $T(0,1)=(0,1), T(1,1)=(0,1)$ and $T(1,0)$ $=(1,0)$.

It is easily checked that $T$ is a continuous and nondecreasing operator (notice that (0, $1) \leq(1,1)$ and $T(0,1)=(0,1) \leq T(1,1)=(0,1))$.

Moreover, as the unique pair of elements in $X$ satisfying $x<y$ is $((0,1),(1,1))$ and $d(T$ $(0,1), T(1,1))=d((0,1),(0,1))=0$, condition (??) of Theorem 2.3 is satisfied.

As $(0,1) \leq T(0,1)$, Theorem 2.3 says us that $T$ has a fixed point (in this case $(0,1)$ and $(1,0)$ are fixed points of $T)$.

On the other hand, the operator $T$ does not satisfy Meir-Keeler condition appearing in

Theorem 1.1 because for $\varepsilon=d_{2}((0,1),(1,0))=\sqrt{2}$ the inequality

$$
d_{2}(T(0,1), T(1,0))=d_{2}((0,1),(1,0))=\sqrt{2}<\varepsilon
$$

fails.

Thus, this example cannot be studied by Meir-Keeler Theorem (Theorem 1.1).

Acknowledgements

Partially supported by Ministerio de Ciencia y Tecnología, project MTM 2007-65706.

Authors' contributions

The three authors have contributed equally in this paper. They read and approval the final manuscript.

Competing interests

The authors declare that they have no competing interests.

Received: 30 June 2011 Accepted: 23 November 2011 Published: 23 November 2011

References

1. Meir, A, Keeler, E: A theorem on contraction mappings. J Math Anal Appl. 28, 326-329 (1969). doi:10.1016/0022-247X (69) $90031-6$

2. Agarwal, RP, El-Gebeily, MA, O'Regan, D: Generalized contractions in partially ordered metric spaces. Appl Anal. 87, 109-116 (2008), doi:10.1080/00036810701556151

3. Altun, I, Simsek, H: Some fixed point theorems on ordered metric spaces and applications. Fixed Point Theory Appl. 2010(621469), 17 (2010)

4. Altum, I, Durmaz, G: Some fixed point theorems on ordered cone metric spaces. Rendiconti del Circolo Matematico di Palermo. 58, 319-325 (2009). doi:10.1007/s12215-009-0026-y 
5. Ciric, L, Cakid, N, Rajovic, M, Ume, JS: Monotone generalized nonlinear contractions in partially ordered metric spaces. Fixed Point Theory Appl. 2008(131294), 11 (2008)

6. Ciric, L, Samet, B, Vetro, C, Abbas, M: Fixed point results for weak contractive mappings in ordered k-metric spaces. Fixed Point Theory. (in press)

7. Ciric, L, Samet, B, Aydi, H, Vetro, C: Common fixed points of generalized contractions on partial metric spaces and an application. Appl Math Comput. 218(6), 2398-2406 (2011). doi:10.1016/j.amc.2011.07.005

8. Gnana Bhaskar, T, Lakshmikantham, V: Fixed point theorems in partially ordered metric spaces and applications. Nonlinear Anal. 65, 1379-1393 (2006). doi:10.1016/j.na.2005.10.017

9. Harjani, J, Sadarangani, K: Fixed point theorems for weakly contractive mappings in partially ordered sets. Nonlinear Anal. 71, 3403-3410 (2009). doi:10.1016/..na.2009.01.240

10. Harjani, J, López, B, Sadarangani, K: Fixed point theorems for weakly C-contractive mappings in ordered metric spaces. Comput. Math Appl. 61, 790-796 (2011)

11. Lakshmikantham, V, Ciric, L: Coupled fixed point theorems for nonlinear contractions in partially ordered metric spaces. Nonlinear Anal. 70, 4341-4349 (2009). doi:10.1016/j.na.2008.09.020

12. Nashine, HK, Samet, B, Vetro, C: Monotone generalized nonlinear contractions and fixed point theorems in ordered metric spaces. Math Comput Modelling. 54, 712-720 (2011). doi:10.1016/..mcm.2011.03.014

13. Nicolae, A, O'Regan, D, Petrusel, A: Fixed point theorems for singlevalued and multivalued generalized contractions in metric spaces endowed with a graph. Georgian Math J. 18, 307-327 (2011)

14. Nieto, JJ, Pouso, RL, Rodríguez-López, R: Fixed point theorems in ordered abstract spaces. Proc Am Math Soc. 135(8), 2505-2517 (2007). doi:10.1090/50002-9939-07-08729-1

15. Nieto, JJ, Rodríguez-López, R: Contractive mapping theorems in partially ordered sets and applications to ordinary differential equations. Order. 22, 223-239 (2005). doi:10.1007/s11083-005-9018-5

16. Nieto, JJ, Rodríguez-López, R: Existence and uniqueness of fixed point in partially ordered sets and applications to ordinary differential equations. Acta Math Sinica. 23, 2205-2212 (2007). doi:10.1007/s10114-005-0769-0

17. O'Regan, D, Petrusel, A: Fixed point theorems for generalized contractions in ordered metric spaces. J Math Anal Appl. 341, 1241-1252 (2008). doi:10.1016/j.jmaa.2007.11.026

18. Petrusel, A, Rus, IA: Fixed point theorems in ordered L-spaces. Proc Am Math Soc. 134(2), 411-418 (2006)

19. Ran, ACM, Reurings, MCB: A fixed point theorem in partially ordered sets and some applications to matrix equations. Proc Am Math Soc. 132, 1435-1443 (2004). doi:10.1090/50002-9939-03-07220-4

20. Sannet, B, Vetro, C: Coupled fixed point theorems for multivalued nonlinear contraction mappings in partially ordered metric spaces. Nonlinear Anal. 74, 4260-4268 (2011). doi:10.1016/j.na.2011.04.007

doi:10.1186/1687-1812-2011-83

Cite this article as: Harjani et al:: A fixed point theorem for Meir-Keeler contractions in ordered metric spaces.

Fixed Point Theory and Applications 2011 2011:83.

\section{Submit your manuscript to a SpringerOpen ${ }^{\odot}$ journal and benefit from:}

- Convenient online submission

- Rigorous peer review

- Immediate publication on acceptance

- Open access: articles freely available online

- High visibility within the field

- Retaining the copyright to your article

Submit your next manuscript at $\boldsymbol{\triangleright}$ springeropen.com 